\title{
Management of Irregular Astigmatism Secondary to Pterygium Removal with the Use of Scleral Contact Lenses
}

\section{Kriti B*}

Cornea and Specialty Contact Lens Service, The Eye Institute at Salus University, USA

*Corresponding author: Kriti Bhagat, Cornea and Specialty Contact Lens Service, The Eye Institute at Salus University, 1200 West Godfrey Ave., Philadelphia, PA 19141, USA, Tel: (215) 276-6000; Email: kbhagat@salus.edu

\section{Case Report}

Volume 3 Issue 3

Received Date: April 14, 2018

Published Date: May 09, 2018

\section{Abstract}

A pterygium is a common ocular surface disorder. Management techniques have been dated back to 1000 B.C., from bile and urine to anti-VEGF injections. Unfortunately, pterygia lead to an irregular surface causing unwanted side effects, such as poor cosmesis, decreased visual acuity (VA), disruption of the tear film and foreign body sensation. Current management strategies for pterygia involve surgical excision, which improves the condition of the ocular surface but does not resolve the irregular astigmatism. This case report outlines a discussion of a patient with a history of bilateral, nasal pterygium removal that had remaining irregular astigmatism decreasing the quality of vision. It reviews the successful fitting of a scleral gas permeable contact lens, leading to an improvement in visual acuity, contrast, and ocular comfort.

Keywords: Pterygium; Irregular Astigmatism; Corneal Topography; Surgical Intervention; Scleral Contact Lenses

Abbreviations: VA: Visual Acuity; UV: Ultraviolet; HPV: Human Papilloma Virus; HIV: Human Immunodeficiency Virus; TGF-? : Transforming Growth Factor.

\section{Introduction}

Pterygium, derived from the Greek word pteryx, which means wing, was described by the world's first known ophthalmologist, Susruta, during the ancient times. Pterygia are wing-shaped, fibrovascular conjunctival lesions extending from the bulbar conjunctiva onto the cornea [1-4]. Historically, pterygia were believed to be a degenerative lesion, a breakdown of Bowman's layer and elastosis [3]. Currently, a pterygium is characterized as a proliferative disorder [5]. It is a centripetally directed growth of squamous and limbal epithelium, goblet cell hyperplasia, underlying stroma with proliferating fibroblasts, neovascularizing and inflammatory cells, and extracellular matrix. A pterygium has three parts - the head, neck, and body [5,1]. The head is the apex enclosed by and a vascular cap, followed by the neck in the region between the head and the corneal limbus. The body of a pterygium is the portion overlying the sclera. During clinical observation, grey lesions adjacent to the cap can be seen. Usually found in advanced cases, these lesions are known as Fuch's flecks, non-proliferating, primitive cells clusters. Iron deposits can also be found, along the apex of the pterygium, forming "Stocker's line" [5]. 


\section{Open Access Journal of Ophthalmology}

Differential diagnosis includes pinguecula, dellen, conjunctival tumors, and pseudopterygium. Pseudopterygia are very similar in appearance but are caused secondary to trauma, surgery, and/or chronic inflammation. They have a broad head, not adherent to the cornea and can grow in any location around the cornea, not limited to 3 and 9 o'clock. Pterygia are predominantly located at the nasal limbus due to peripheral lighting focus on the nasal limbus, although temporal pterygia are also common. Patients with pterygia can be found throughout the globe, higher incidences reported in those living closer to the equator. Extensive studies have shown a strong correlation in pterygia findings in patients with more UV (ultraviolet) exposure, leading to the belief that those in periequatorial latitudes are of higher risk. Recent studies show Eskimos and sailors to be at a higher risk due to snow and water reflecting UV light. As summarized in Table 1, the long list of risk factors includes cumulative UV exposure, geographic location, age, occupation, family history, and HPV (Human Papilloma Virus) /HIV (Human Immunodeficiency Virus) association [3].

\begin{tabular}{|c|}
\hline Risk Factors \\
\hline Location - close to equator \\
\hline Hot, dry, dusty climate \\
\hline Age \\
\hline Family History \\
\hline Cumulative UV exposure \\
\hline Male \\
\hline Rural residency \\
\hline Welders, laborers, and outdoor workers \\
\hline Sawmill workers \\
\hline HPV/HIV association \\
\hline
\end{tabular}

Table 1: Risk factors for Pterygia.

The pathogenesis of pterygia consists of two mechanisms; environmental (UV exposure, viral) and/or growth promoting markers (cytokines, MMP). Each risk factor plays an integral role in the creation and progression of the pterygium [3]. The role of stem cells has been debated through many studies. In one study, chronic exposure to UV light is believed to activate limbal stem cells that cause clonal expansion. Yet other studies display signs of focal limbal stem cell deficiency, leading to conjunctival in growth and corneal vascularization. Together, the theories speculate it may be a dysfunction of the limbal stem cells causing a combination of expression and inactivity. Aberrant TGF- $\beta$ signaling is thought to contribute to progressive fibrosis in pterygia. Association with HPV is believed due to its ability to inactivate the p53 gene leading to tumor-like cells found in pterygia. Analysis of DNA shows damage such as loss of heterozygosity, microsatellite instability, and mutations in Kirsten-ras and p53 genes. Studies have shown damage to inhibitor proteins that control cell cycle progression, leading to uncontrolled cell division, leading to apoptosis and DNA leakage into the fibrovascular layer [3]. Other theories include mesenchymal transition causing formation of fibroblasts, bone-marrow-derived progenitor cells and neurogenic inflammation. ${ }^{3}$

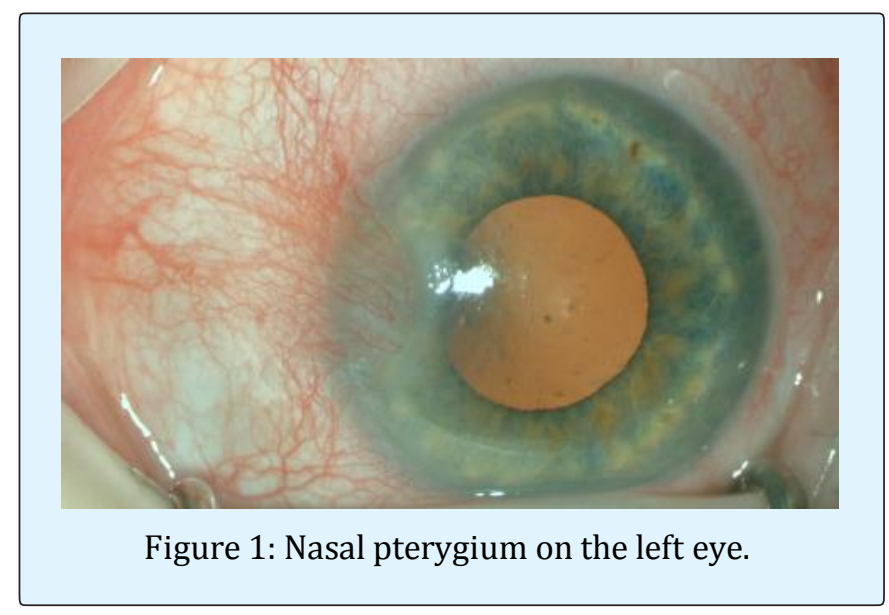

The goal of treating pterygia is to restore ocular surface anatomy, restore or improve function, improve cosmesis, and alleviate all associated symptoms. Preventing the formation or progression of pterygia is by decreasing UV exposure. Eye protection by wraparound sunglasses and wide brimmed hats should be stressed to everyone [3]. For those who have already been diagnosed, they should be monitored with yearly serial photography. Medical management options include lubricants, antiinflammatory topical agents such as NSAIDs or steroids. More recent strategies include cyclosporine $0.05 \%$ as a safe treatment for long-term anti-inflammatory treatment, while co-managing dry eye syndrome symptoms that can be caused by pterygia. Current studies are being done to evaluate the efficacy of oral doxycycline, as it serves as an inhibitor of the pathogenesis cascade. Due to irregular corneal changes caused by the pterygium, significant astigmatism may be induced [3]. In less severe cases, the with-the-rule astigmatism or against-the-rule astigmatism can be managed with spectacles or soft toric contact lenses. In advanced cases, the irregular corneal astigmatism can be managed with other specialty contact lens options. Pterygia may require surgical management 


\section{Open Access Journal of Ophthalmology}

in cases of discomfort, cosmesis, or obstruction of vision. The goal of pterygium treatment is avoiding recurrence, but complication rates and cosmetic results are also weighed [6].

The gold standard for surgical management of pterygia is excision with the use of autoconjunctival reconstructive grafting. This management provides a low recurrence and complication rate. Recent studies have shown a decrease in the already reduced rate by using fibrin glue instead of sutures. Alternatives to fibrin glue include never techniques, such as autologous blood and electrocautery pen, yet both need larger clinical trials. A related procedure is the limbal conjunctival autograft, in which limbal tissue is included in the graft source and then transferred to the destination [6]. It is hypothesized that this will prevent the residual tissue from proliferating with faster healing with the addition of limbal stem cells [6]. Although limbalautografts have a lower recurrence rate in comparison to conjunctival grafts, the complications are more severe and the procedure takes longer. Another adjunct therapy commonly used after a bare scleral excision is to cover it with an amniotic membrane. Amniotic membrane has antinflammatory properties as it promotes epithelial growth and suppresses transforming growth factor (TGF- $\beta$ ) signaling fibroblast suppression. Amniotic membrane recurrence rates are high compared to conjunctival autografts, although newer techniques with steroid injections have reported lower recurrence rates. Other newer trends include the use of cyclosporine post-operatively to reduce inflammation, mitomycin C (MMC), and beta radiation to modify wound healing, as well as antiVEGF/antiangiogenic therapy to induce vessel regression in pterygia. All excised pterygia should be submitted for histopathology examination due to $10-30 \%$ of specimens having unsuspected neoplastic cellular findings [3].

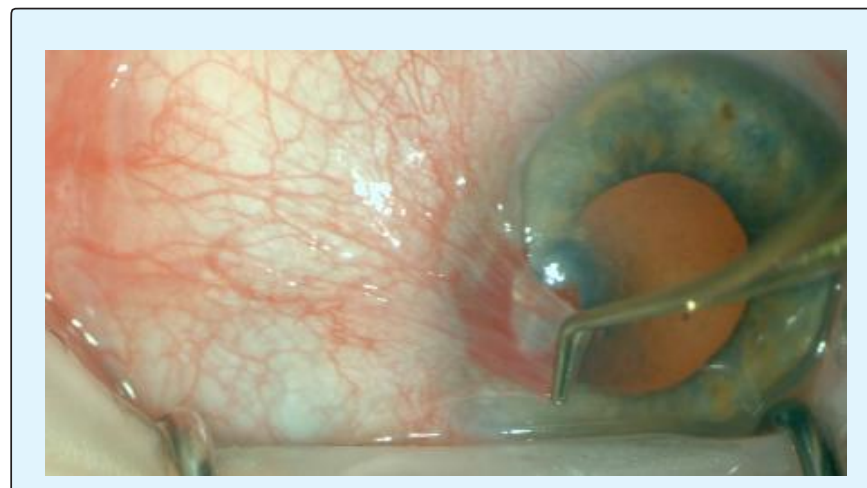

Figure 2: Preparation of surgical excision.

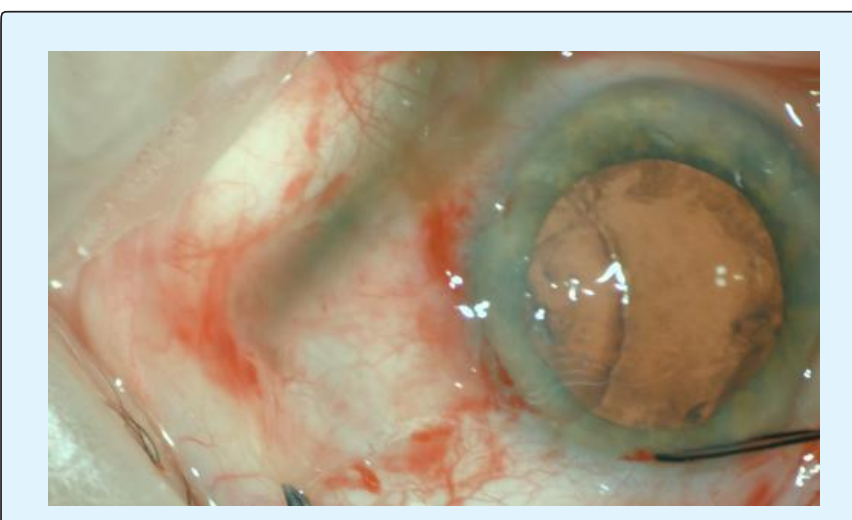

Figure 3: Conjunctival autograft overlying excised pterygium tissue

Surgical removal of pterygia can improve the ocular surface leading to decreased discomfort, irritation, and a reduction in induced astigmatism. Improving the induced astigmatism does not normalize the cornea; irregular topographic findings will persist. Yaycioglu, et al. found that there was a positive correlation between the preoperative size of the pterygium and the astigmatism degree. This also correlates to a greater change in astigmatism after surgical intervention. They also found that the method of surgery and type of grafting did not have a significant effect on the amount of astigmatic change [8]. This suggests that surgeons choose grafting techniques based on recurrence rate as opposed to refractive results. Although an increase in visual acuity (VA) is expected following surgical excision, corneal irregularity remains. Surgical excision is also a carefully made decision, weighing the benefits versus complications, as it is one of the most painful ophthalmic procedures.

This case report illustrates the management of a patient who underwent bilateral, nasal pterygium removal. For years, he maintained "20/20" vision in each eye, ignoring the decreased contrast and peripheral aberrations. The case will examine how a scleral contact lens provided a significant increase in the quality of vision.

\section{Case Report}

A 65 -year-old African American male was referred to the specialty contact lens clinic for a fitting. His chief complaint was decreased visual acuity and difficulty adjusting to a new pair of progressive spectacle glasses. The patient reported having bilateral, nasal pterygium excisions in the early 2000s. No further information on 


\section{Open Access Journal of Ophthalmology}

the type of grafting, size of pterygium, or previous history was obtainable. He reported that several doctors had attempted to fit him with "hard" lenses. Despite an improvement in visual acuity, the discomfort caused him to discontinue wear. He was referred for a "scleral" lens fitting after a recent visit to his corneal ophthalmologist. The suggestion was to "fit in a safer gas permeable lenses that would not only irrigate the cornea and conjunctiva while improving the quality of vision". The patient's medical history was unremarkable. He denied taking any medications and had no known drug allergies.
While wearing his spectacles, the patient's entering visual acuity was $20 / 25$ right eye (OD) and 20/40 left eye (OS). Corneal evaluation revealed nasal thinning and scarring with irregularity of the reflecting tear film in both eyes. Nasal corneas also revealed a brown line outlying the inner edge of the nasal scarring. This is Stocker's line, a common finding of iron deposition found along the head of the pterygium. Corneal scarring spares the pupillary axis in both eyes, which allows the patient to be corrected in either spectacles or contact lenses. Entrance testing also included a corneal topography.

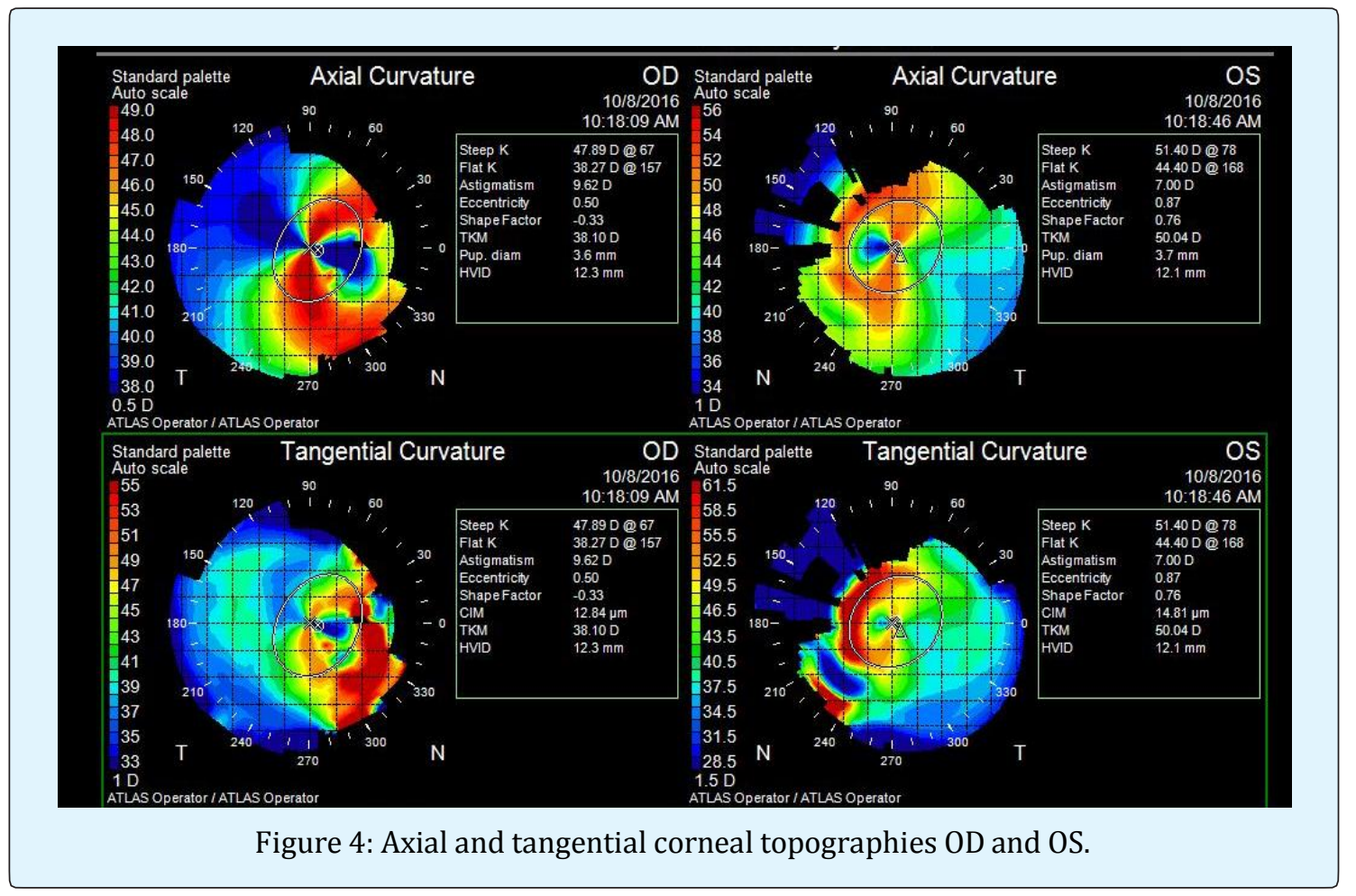

The topographical corneal map revealed key elements of the amount and location of the corneal irregularity. At first glance, the topographies of both eyes reveal a nasal pellucid marginal degeneration like pattern, which is immediately ruled out through the patient's history. Both eyes have irregular nasal steepening surrounding a flatter curvature. Although the right eye has a higher amount of corneal cylinder, -9.46 diopters, versus the left eye, -6.62 diopters; the left eye has a greater amount of corneal eccentricity.

After examining his previous history, goals for increasing the quality of vision, and entrance testing, it was determined that the patient would best be fit in a scleral contact lens. After two visits, the patient was fit successfully with customized scleral lenses.

Insertion and removal training was successfully completed at this visit. The patient was given samples of solutions, insertion and removal devices (including large DMV, small DMV, and a scleral lens inserter ring), and written instructions. The patient was instructed to care for lenses by using a hydrogen peroxide based solution. He was instructed to fill the bowl of the lens with a nonpreserved saline solution. With in-office training and written instructions reviewed, the patient was advised to start a gradual increase in wear time until a full day. It was strongly stressed to never sleep, shower, or swim with the lenses. The patient expressed comfort with 


\section{Open Access Journal of Ophthalmology}

insertion and removal of the lenses, so the lenses were dispensed and a follow-up visit to evaluate the fit of the lens was made.

\section{Visit 3}

After using the lenses for 2 weeks, the patient returned for his final follow-up. He arrived wearing the lenses for six hours without having any complications and in good comfort. He reported ease with inserting and removing lenses, and being able to read every day, common material. The patient reported an increase in contrast and color with everyday objects. This was the greatest change he had experienced and "felt life-changing".

With an excellent fit, optimal visual acuity, and great comfort, the lens fit was finalized. Contact lens hygiene and care was reviewed. The patient was advised to immediately call and remove lenses if any redness or irritation developed. The patient made an appointment in 6 months to re-evaluate the fit, and he will continue annual comprehensive exams with his corneal specialist.

\section{Discussion}

Contact lens use for refractive indications has been used since 1845. As the lenses have developed, so has the use of contact lens in cases of corneal irregularities [9]. Contact lenses have done a full 360 as the first lenses developed were scleral lenses in 1888. The purpose was to neutralize the optical effects of the distortion in patients with irregular astigmatism. Although the glass shell provided a tremendous improvement in vision during that time, the complications outweighed the benefits. Through this failed attempt, other lenses were pioneered leading to the resurrection of scleral lens use in recent years.

Irregular corneal astigmatism is defined as astigmatism where the principal meridians are not 90 degrees a part [9]. Causes for irregular astigmatism can range from corneal degenerations to infectious by-products to inflammatory processes. Ocular surface disease, keratoconus, pellucid marginal degenerations, corneal scarring, pterygia and post-refractive surgical corneas are all examples in which the corneal surface can be irregular. Most commonly, irregular astigmatism decreases the visual acuity but in some cases, the visual acuity can still be $20 / 20$ and the quality of the vision is deteriorated. Tomidokoro, et al. found that corneal irregularity caused by refractive surgery can significantly decrease contrast sensitivity. Other theories have shown an increase in glare visual acuity in patients with irregular astigmatism.
Corneal irregularity can disrupt the tear film leading to tear film instability, possibly causing dry eye syndrome. Due to the conforming nature of soft contact lenses, patients with irregular corneas are unsuccessful with mass-produced soft contact lenses.

Corneal topography is an essential diagnostic tool when evaluating patients with an irregular corneal surface. The topographer is a non-invasive imaging tool used to map the reflective surface curvature of the cornea. Wave front analysis can be used to predict higher-order aberrations, explaining why irregular astigmatism patients can be $20 / 20$ but intolerant of their spectacle glasses, as in the case of our patient.

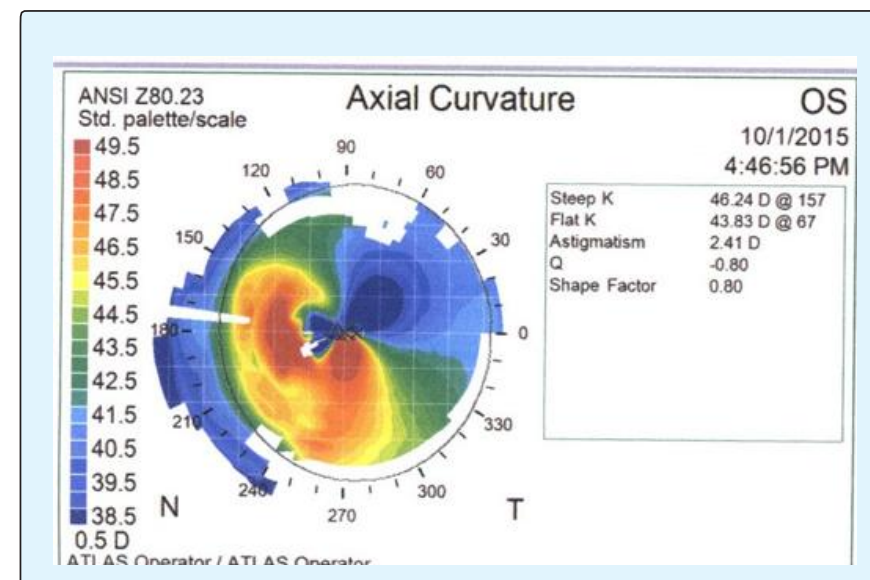

Figure 5: Example of an axial topography of a patient with a nasal pterygium.

Due to the patient's previous history of bilateral pterygia, it is important to understand the relationship between pterygia and astigmatism. In patients with pterygia, corneal astigmatism can be a combination of naturally occurring astigmatism and astigmatism induced by the pterygium. Pterygia are believed to cause corneal distortion through two possible mechanisms [4]. One proposed mechanism is the tractional forces of contractile elements within the pterygium mechanically distorting and flattening the cornea. The second mechanism involves the localized pooling of tears at the pterygium apex leading to an observation of corneal flattening by the topographer. Pterygia generally cause with-the-rule corneal astigmatism due to localized flattening towards the apex of the pterygium [4]. Mohammad-Salih et al. found correlations between the size of the pterygium and the amount of corneal astigmatism. They found a significant correlation between an increases in pterygium extension onto the cornea, increased the amount of 


\section{Open Access Journal of Ophthalmology}

induced astigmatism [11]. Since the size (width, extension, and area) can be a strong predictor of the induced astigmatism, this can also be seen as an indicator of surgical intervention, decreasing corneal distortion and visual disturbances.

Surgical excision generally causes astigmatism and topographic irregularity to improve, leading to an improvement in visual acuity. Similar to previous studies, the degree of astigmatism reduction is related to the preoperative size of the pterygium [6]. Improving the amount of corneal astigmatism has shown to improve visual acuity. Surgical excision has shown improvement in the amount of corneal astigmatism but, unfortunately, the type of grafting does not have significant effect [8]. Tomidokoro, et al. summarizes by stating that surgical excision improves corneal astigmatism, but in advanced cases, does not completely normalize corneal distortion and irregularity.

Persistent corneal irregularity and distortion in postsurgical pterygium removal is what this patient presented with. Spectacle correction would be the first line of treatment due to safety in a post-surgical patient [8]. The patient was correctable to a functional visual acuity, allowing him to perform activities of daily living, including driving, but still reported discomfort with spectacles due to distortions. Wave front guided surgical treatment would decrease the aberrations but would be contraindicated due to previous corneal pathology and surgical intervention. Penetrating keratoplasty would be another method to decrease, not resolve, optical aberrations leading from the corneal irregularity. This option is not indicated due to a clear, non-infectious cornea therefore the complications would outweigh possible benefits. The safest and most beneficial option to treat this patient would be a type of specialty contact lens.

Post-pterygium removal corneas can rarely be fit with soft toric lenses. In mild irregularity cases, this may be an option but due to peripheral eccentricity and higher order aberrations, the soft lens may not provide good visual quality. In cases of irregular astigmatism, rigid gas permeable lenses have been shown to improve the visual acuity and quality. With an aligned fit, the concept is to create a regular refracting layer by the tear layer filling the space between the contact lens and cornea [7]. Another alternative to smaller diameter rigid gas permeable lenses may be hybrid lenses, a more comfortable alternative that provides equal vision improvement. The central gas permeable area corrects for irregular astigmatism while the soft skirt provides good comfort. With a similar concept and newer technology, there is a new option in the world of specialty contact lenses, the scleral lens.

Scleral lenses provide a beneficial alternative option, whether it improves comfort, improves the fitting relationship between a contact lens and the cornea, and/or creates a safe environment for an already compromised cornea. These lenses are generally larger in size (14 $\mathrm{mm}$ to $20 \mathrm{~mm}$ ), which can be very advantageous for the patient and the cornea. The landing zone of these lenses is on the less innervated sclera, leading to great comfort. Fortunately, the patient did not have signs of lens edge lift or impingement into the sclera at the landing zone. Toric peripheral curves can be designed in scleral lenses, in cases where one meridian may be flatter or steeper than the other. If conjunctival vessel impingement is observed, a toric peripheral landing is advised to evenly distribute the weight of the lens. Similar to a rigid gas permeable lens, a tear lens (non-preserved saline), is created between the contact lens and the cornea creating a regular, refracting surface [2]. Scleral lenses are generally fit based off of the sagittal depth, therefore higher amounts of cornea irregularity (i.e. advanced keratoconus) can be successfully fit. In the case of our patient, with irregular steepening only in the nasal meridian, it is hard to create a steepening effect only in one quadrant with an RGP without dislocation. A properly fit scleral, as the lens that was designed, can vault over the irregularity while providing great visual acuity. In recent years, scleral lenses have served to improve mainly refractive conditions, yet more research is being conducted on their therapeutic advantages.

In this case, scleral lenses were an ideal solution for the patient [9]. He had expressed that he had attempted "hard" lenses when he was younger and did not want to pursue wearing them again. Unlike the other lens options, scleral lenses are traditionally costlier, from the fitting fee to the cost of the lenses, which proved immaterial to this patient. From the beginning, the patient had expressed interest in being able to see distance and near, which was provided using the chosen lens design. Scleral contact lenses require multiple solutions: non-preserved saline to fill the lens, a daily cleaner for gas permeable material, and a stronger cleaner for long term protein build up. Incorrect use of the recommended solutions can cause multiple complications. The use of multiple solutions can also be inconvenient for patients leading to noncompliance and causing complications. Insertion and removal techniques for scleral lenses can be difficult, especially for the elderly or those who may be limited in 


\section{Open Access Journal of Ophthalmology}

their mobility. When fitting patients with scleral lenses, an assessment of how the patient will handle the lenses should be reviewed before recommending these lenses.

\section{Conclusion}

Despite public education on preventative care against UV light damage, pterygia are a common finding in an optometric practice. Managing patients with induced irregular astigmatism induced by pterygia or their excision can be done through the use of various specialty contact lenses. Modalities like scleral lenses manage induced astigmatism from surgical intervention with minimal side effects while providing great visual acuity potential, as seen in this case. Surgical excision is recommended earlier in pterygia removal to reduce overall induced astigmatism. Practitioners should be familiar with all lens options available to improve the final outcome. The strongest point made through this case was not the improvement in visual acuity or great comfort with scleral lenses, it was the patient expressing a tremendous increase in contrast and improving the overall quality of vision. It's important to consider high order aberrations in patients with irregular astigmatism, which can be managed with specialty contact lenses like scleral lenses.

\section{References}

1. Fuest M, Liu YC, Yam GH, Teo EP, Htoon HM, et al. (2017) Femtosecond laser-assisted conjunctival autograft preparation for pterygiumsurgery. The Ocular Surface 15(2): 211-217.

2. Kaufman SC, Jacobs DS, Lee WB, Deng SX, Rosenblatt MI, et al. (2013) Options and adjuvants in surgery for pterygium: a report by the American Academy of Ophthalmology. Ophthalmology 120(1): 201-208.
3. Coroneo M, Chui J (2013) Pterygium. In Ocular Surface Disease. Elsevier pp: 125-137.

4. Mohammad Salih PA, Sharif AF (2008) Analysis of pterygium size and induced corneal astigmatism. Clinical Science 27(4): 434-438.

5. Chui J, Coroneo MT, Tat LT, Crouch R, Wakefield D, et al. (2011) Ophthalmic pterygium: a stem cell disorder with premalignant features. The American Journal of Pathology 178(2): 817-827.

6. Janson B, Sikder S (2014) Surgical management of pterygium. The Ocular Surface 12(2): 112-119.

7. Kalwerisky K, Davies B, Mihora L, Czyz CN, Foster JA (2011) Use of the boston ocular surface prosthesis in the management of severe periorbital thermal injuries. Ophthalmology 119(3): 516-521.

8. Altan-Yaycioglu R, Kucukerdonmez C, Karalezli A, Corak F, Akova Y (2013) Astigmatic changes following pterygium removal: comparison of 5 different methods. Indian Journal of Ophthalmology 61(3): 104-108.

9. Romero-Rangel T, Stavrou P, Cotter J, Rosenthal P, Baltatzis S (2000) Gas-permeable scleral contact lens therapy in ocular surface disease. American Journal of Ophthalmology 130(1): 25-32.

10. Tomidokoro A, Oshika T, Amano S, Eguchi K, Eguchi S (2009) Quantitative analysis of regular and irregular astigmatism induced by pterygium. Cornea 18(4): 412-415.

11. Mohammad-Salih, Payman AK, Sharif AF (2008) Analysis of pterygium size and induced corneal astigmatism. Cornea 27(4): 434-438. 